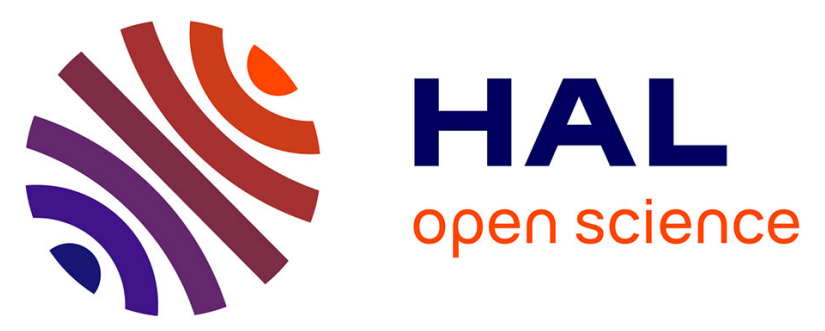

\title{
A physiologically based pharmacokinetic model of vascular-extravascular exchanges during liver carcinogenesis: application to MRI contrast agents.
}

Muriel Mescam, Pierre-Antoine Eliat, Claire Fauvel, Jacques D. de Certaines, Johanne Bézy-Wendling

\section{To cite this version:}

Muriel Mescam, Pierre-Antoine Eliat, Claire Fauvel, Jacques D. de Certaines, Johanne Bézy-Wendling. A physiologically based pharmacokinetic model of vascular-extravascular exchanges during liver carcinogenesis: application to MRI contrast agents.. Contrast Media and Molecular Imaging, 2007, 2 (5), pp.215-28. 10.1002/cmmi.147 . inserm-00174336

\section{HAL Id: inserm-00174336 https://www.hal.inserm.fr/inserm-00174336}

Submitted on 25 Sep 2007

HAL is a multi-disciplinary open access archive for the deposit and dissemination of scientific research documents, whether they are published or not. The documents may come from teaching and research institutions in France or abroad, or from public or private research centers.
L'archive ouverte pluridisciplinaire HAL, est destinée au dépôt et à la diffusion de documents scientifiques de niveau recherche, publiés ou non, émanant des établissements d'enseignement et de recherche français ou étrangers, des laboratoires publics ou privés. 


\section{A Physiologically Based PharmacoKinetic model of} vascular-extravascular exchanges during liver carcinogenesis: application to MRI contrast agents

\section{A PBPK model of liver microvascularization}

Muriel Mescam ${ }^{1,2, *}$, Pierre-Antoine Eliat ${ }^{3}$, Claire Fauvel ${ }^{1,2,3}$, Jacques D. de Certaines ${ }^{3,4}$, Johanne Bézy-Wendling ${ }^{1,2}$

1 INSERM, U642, Rennes, F-35000, France;

2 Université de Rennes 1, LTSI, Rennes, F-35000, France;

${ }^{3}$ PRISM - ImagiVeC UPRES-EA 3890 - IFR 140 GFAS, OUEST-génopole ${ }^{\circledR}$, Faculté de Médecine, Université de Rennes 1;

${ }^{4}$ Centre Eugène Marquis, Département de Biologie, Rennes.

\footnotetext{
${ }^{*}$ Corresponding author: M. Mescam, LTSI, Campus de Beaulieu, Université de Rennes 1, 263 Avenue du Général Leclerc - CS 74205 - 35042 Rennes Cedex, France, Tel:(33)223236220, Fax:(33)223236917, E-mail: muriel.mescam@univ-rennes1.fr
} 


\begin{abstract}
The extraction of physiological parameters by non-invasive imaging techniques such as dynamic magnetic resonance imaging (MRI) or positron emission tomography requires a knowledge of molecular distribution and exchange between microvascularization and extravascular tissues. These phenomena not only depend on the physicochemical characteristics of the injected molecules but also the pathophysiological state of the targeted organ. We developed a five-compartment physiologically based pharmacokinetic model focused on hepatic carcinogenesis and MRI contrast agents. This model includes physical characteristics of the contrast agent, dual specific liver supply, micro-vessel wall properties and transport parameters that are compatible with hepatocarcinoma development. The evolution of concentrations in the five compartments showed significant differences in the distribution of three molecules (differentiated by their diameters and diffusion coefficients ranging, respectively, from $0.9 \mathrm{~nm}$ to $62 \mathrm{~nm}$ and from $68 \cdot 10^{-9} \mathrm{~cm}^{2} \cdot \mathrm{s}^{-1}$ to $47 \cdot 10^{-7} \mathrm{~cm}^{2} \cdot \mathrm{s}^{-1}$ ) in simulated regeneration nodules and dysplastic nodules, as well as in mediumand poorly-differentiated hepatocarcinoma. These results are in agreement with known vascular modifications such as arterialization that occur during hepatocarcinogenesis. This model can be used to study the pharmacokinetics of contrast agents and consequently to extract parameters that are characteristic of the tumor development (like permeability), after fitting simulated to in-vivo data.
\end{abstract}

KEYWORDS: PBPK model, molecule transport, liver microvascularization, contrast agent, cancer, HepatoCellular Carcinoma, MRI, simulation

Abbreviations used: MRI, Magnetic Resonance Imaging; DCE, Dynamic ContrastEnhanced; PBPK, Physiologically Based PharmacoKinetic; HCC, HepatoCellular Carcinoma; CT, Computed Tomography; US, UltraSound; RN, Regenerative Nodule; MID, Multiple Indicator Dilution; PET, Positron Emission Tomography; Gd-DOTA, Gadolinium -(1,4,7,10-tetraazacyclododecane N,N',N",N"' -tetraacetic 
acid); SPIO, SuperParamagnetic Iron Oxide; USPIO, UltraSmall Particle of Iron Oxide; PCHIP, Piecewise Cubic Interpolating Polynomial. 


\section{INTRODUCTION}

Dynamic contrast enhanced (DCE) images are of great interest for the characterization of hepatic tumors such as those giving rise to Hepatocellular Carcinoma (HCC). Such images can be acquired by the main techniques used in routine clinical studies (Magnetic Resonance Imaging (MRI), Computed Tomography (CT) and Ultrasound (US)) [1]. DCE imaging during and after injection of a contrast agent improves the characterization of lesions because it allows us to access morphological and functional features that are characteristic of the tumor development [2]. In addition to in vivo studies [3], in silico modeling is a means of studying the behavior of different kinds of molecules. More particularly, this technique is useful to improve our knowledge on the distribution of the numerous contrast agents already available [4]. In addition, it can be used to predict the distribution of new agents, in order to optimize the image acquisition (type of agent, injection method, type of acquisition involving sequence in MRI and acquisition times). The objective of such optimization is to strive for an early characterization of the lesion, by determining its grade in the complex carcinogenesis process in a cirrhotic liver, which corresponds to the progressive malignant transformation of a benign Regenerative Nodule $(\mathrm{RN})$ into a malignant tumor, HepatoCarcinoma (HCC) [5] (Fig.1). The Regenerative Nodule is made up of normal hepatic cells, associated with an architectural disruption. Its vascular portal and arterial supplies are similar to those of the adjacent normal parenchyma. During the progressive malignant transformation of the nodule, we observe a rarefaction of the portal tracts (structures containing the hepatic arteriole and portal venule), together with an arterial neoangiogenesis (hatched gray region on the diagram). These new vessels have morphological and physiological characteristics that differ from a normal hepatic arteriole (in gray on figure 1). The portal supply of the nodule decreases progressively until it is canceled out in the poorly-differentiated HCC (HCCp). The phenomena are slightly more complex re-

garding the arterial supply (gray zone plus the hatched gray region corresponding to 
neo-vessels): an initial decrease of flow is observed in low-grade dysplastic nodules $(\mathrm{DNl})$, which then progressively increases due to the arterial neovascularization. For high-grade dysplastic nodules (DNh) and well-differentiated $\mathrm{HCC}(\mathrm{HCCw})$, the arterial flow growth, due to neo-vessels, can fully compensate for the drop in flow of the normal arteriole.

Distribution of solutes in the whole liver has been widely studied by multiple indicator dilution (MID), wich consists in studying simultaneously several tracers with different behavior, mostly for experimental measurements such as vascular and extravascular blood volumes [6] [7] [8] [9]. Several other studies, that can be considered as "global", have been carried out to model the transport of molecules. For example, we can cite a number of adaptations of the one-tissue compartment model based on a Kety-Schmidt type approach and applied to PET images [10] [11] [12] [13] [14] [15]. Materne et al. [16] [2] proposed a dual-input (aorta and portal vein) one-compartmental model to quantify liver perfusion. Although this model is well adapted to hepatic microcirculation, the approach is global since the whole liver is considered as only one compartment. Several other global studies were performed concerning the whole liver but still not at the microscopic scale [17] [18] [19].

In earlier work, Bert \& Pinder [20] developed a three-compartment model of the human microvascular system that integrated fluid mechanics with protein and flow variations. Although this model simulates capillary fluid movements precisely, it is not adapted to hepatic microvascularization because the portal supply is not taken into account. With this aim in view, many works were applied to the liver [22] [21] [23]. However, they do not integrate physiological or geometrical parameters. In 2004, Bézy-Wendling and Kretowski integrated a three-compartment model of hepatic microvascularization into a macroscopic model of the hepatic vascular system in order to simulate CT images of liver tumors [24] [25]. Although this physiologically-based model uses several parameters such as osmotic and hydrostatic pressures to measure flows, it does not allow for variations in the permeability 
of capillaries and vessels, or changes in molecular properties.

In the present study, we propose a Physiologically Based PharmacoKinetic model that is able to simulate the propagation of contrast agents in the small hepatic vessels, while taking into account the specificity of liver dual supply (arterial and portal) as well as the sinusoids. We incorporate the most important parameters showing variations that characterize the tumoral stage evolution: these are mainly transport parameters (vascular permeability, hydraulic conductivity, reflection coefficient) related to the properties of the molecule and of the vascular walls, but also include the exchange area and pressure gradients. In the methods section, we present the physiological parameters that need to be considered and describe the model in detail. The last section is concerned with evaluation of the properties of the model through simulation results, as well as its application to represent molecular exchanges with three contrast agents - Gd-DOTA (Dotarem ${ }^{\circledR}$, Guerbet, France, $0.9 \mathrm{~nm}$ diameter and $70 \times 10^{-7} \mathrm{~cm}^{2} . \mathrm{s}^{-1}$ diffusion coefficient [26]), P792 blood pool agent (Gadomelitol, Vistarem ${ }^{\circledR}$, Guerbet, France, $5 \mathrm{~nm}$ diameter and $8.4 \times 10^{-7} \mathrm{~cm}^{2} . \mathrm{s}^{-1}$ diffusion coefficient [27]) and SuperParamagnetic Iron Oxide (SPIO) SHU-555-A (Resovist, Shering, Germany, 62nm diameter and $68 \times 10^{-9} \mathrm{~cm}^{2} \cdot \mathrm{s}^{-1}$ diffusion coefficient [28]) - in the case of normal liver, dysplastic nodule and differentiated hepatocellular carcinoma. 


\section{METHODS}

\section{The Model}

We present here a five-compartment model of an hepatic lobule that fully integrates the hepatic arteriole and the portal venule as compartments and not just as simple inputs of the model. The five compartments are then the hepatic arteriole, the portal venule, the sinusoidal network, the hepatic venule and the interstitial fluid. The inputs are the terminal branches of the hepatic artery and the portal vein, which supply the hepatic lobule, considered here as the functional unit of the liver parenchyma (Fig.2). The output is the terminal hepatic venule, supplied by a capillary network that composes the sinusoidal compartment. These compartments do not only communicate with each other while the molecule is propagating, since transmembranar exchanges also exist between some compartments and the interstitial fluid inside the lobule. A model of these exchanges was already applied by Kellen et al. [29] in the cardiovascular system.

An overview of our model is given in Fig.3. In this model, several pathways denoted as $k$, and modelled as "holes" in the vascular walls, are considered. For example, sinusoids are a particular type of discontinuous capillaries that exhibit large fenestrations $(k=f)$, as well as small and large pores $(k=s p$ and $k=l p)$. Another way occurring in tumoral tissue can be taken into account and concerns fluid transport through endothelial cells $(k=e n d o)$. On the contrary, the arteriole and venule are assumed to be impermeable in a normal liver. All parameters used in the following are listed in table 1.

\section{Physiological parameters}

Several parameters determine the exchanges of vascular and extravascular fluids and molecules in a tissue. Some, such as the vascular permeability $P$, the hydraulic conductivity $L$ and the reflection coefficient $\sigma$, are said to be "transport" parameters. 
They depend on physicochemical properties of the molecule (size, charge, structure and fixation) and on physiological and physicochemical properties of the vascular wall (endothelial junctions, number of fenestrations, transendothelial ducts, continuity or discontinuity of the membrane, etc.).

Other important physiological parameters include the surface-area of exchange $A_{k}$, related to the $k^{s t}$ pathway, concentration gradients and pressure gradients. In the case of small molecules, crossing of the vascular wall usually occurs by diffusion and is assisted by concentration gradients. However, when large molecules are concerned (bigger than fenestrations), this occurs via convection owing to pressure gradients. Transmembranar fluid exchanges $J_{f}^{k}$ are controlled by forces that correspond to hydrostatic $(p)$ and osmotic (П) pressures. Hydrostatic pressure arises from gravitational forces, and decreases from the arterio-portal to the venous extremities. On the other hand, osmotic pressure arises from solute concentration gradients, and varies from one side of the capillary wall to the other (Fig.4). However, for the moment, no spatial information is integrated into the model.

The filtration-based fluid movement through a vessel wall is governed by Starling's hypothesis [30], which proposes that this phenomenon depends on an equilibrium between the hydrostatic and osmotic pressure gradients across the wall, as shown in Eq.1.

$$
J_{f}^{k}=L_{k}\left\{\left(p_{v}-p_{i l}\right)-\left[\sigma_{k}\left(\Pi_{v}-\Pi_{i l}\right)\right]+\Pi_{M}\right\}
$$

The subscripts $v$ and ${ }_{i l}$ stand for vessel and interstitial fluid, respectively.

$\Pi_{M}$ defines the osmotic pressure of the immobile interstitial matrix, which is related to the matrix osmotic pressure coefficients $\psi_{n=1,2,3}$, and the quantity of interstitial matrix $M_{i m}$ (Eq.2) [29].

$$
\Pi_{M}=\psi_{1} M_{i m}+\psi_{2} M_{i m}^{2}+\psi_{3} M_{i m}^{3}
$$


The transport of molecules (solute flux $J_{s}^{k}$ ) through the pores of a vessel or a capillary membrane can be described by Patlak's equation with separate diffusive and convective components [30] [31], Eq.3.

$$
J_{s}^{k}=P_{k}\left(C_{v}-C_{i l}\right) \frac{P e_{k}}{e^{P e_{k}}-1}+J_{f}^{k}\left(1-\sigma_{k}\right) C_{v}
$$

The Peclet number Pe, defined in Eq.4, is a dimensionless number that represents the ratio of mass transport by convection to mass transport by diffusion:

$$
P e_{k}=\frac{J_{f}^{k}\left(1-\sigma_{k}\right)}{P_{k}}
$$

Both transport mechanisms (solute and solvent) can be coupled, especially for transit through pores and fenestrations.

$$
\begin{gathered}
J_{f}=J_{f}^{e n d o}+J_{f}^{s p}+J_{f}^{l p}+J_{f}^{f} \\
J_{s}=J_{s}^{s p}+J_{s}^{l p}+J_{s}^{f}
\end{gathered}
$$

However, for most hydrophilic solutes, when endothelial crossing is taken into account, the only process activated is the volume flux of pure water $J_{f}^{\text {endo }}$ (Eq.7), proportionally to the transendothelial hydraulic conductivity $L_{\text {endo }}$ [29].

$$
J_{f}^{\text {endo }}=L_{\text {endo }}\left\{\left(p_{v}-p_{i l}\right)-\left[\left(\Pi_{v}-\Pi_{i l}\right)\right]+\Pi_{M}\right\}
$$

\section{Pore model for transcapillary exchanges}

Three possible pathways $k=s p, l p, f$ are considered for the exchanges of water and solute through the pores of the vessel membrane. Those "idealized" pores are modeled as cylinders that permit spherical solutes to cross the membrane according to Bean's equations, as cited by Kellen [29]. These equations are associated with the hydraulic conductivity $L_{k}$ (Eq.8), the permeability $P_{k}$ (Eq.9) and the reflection 
coefficient $\sigma_{k}$ (Eq.10).

$$
\begin{gathered}
L_{k}=\left(\frac{A_{k}}{S \Delta r}\right) \frac{r_{k}^{2}}{8 \eta} \\
P_{k}=\left(\frac{A_{k}}{S \Delta r}\right)\left(1-\alpha_{k}\right) F\left(\alpha_{k}\right) D \\
\sigma_{k}=1-\left[1-\left(1-\left(1-\alpha_{k}\right)^{2}\right)^{2}\right] G\left(\alpha_{k}\right)+\frac{16}{9} \alpha_{k}^{2}\left(1-\alpha_{k}\right)^{2} F\left(\alpha_{k}\right) \\
\alpha_{k}=\frac{r}{r_{k}}
\end{gathered}
$$

$S$ defines the vascular compartment surface-area. $\eta$ is the perfusate viscosity. The total area of the k-like pores $A_{k}$ in one compartment is obtained from their radius $r_{k}$ and their density $d_{k}$ in that compartment [30] [32]. $\quad F\left(\alpha_{k}\right)$ and $G\left(\alpha_{k}\right)$ are decreasing hydrodynamic functions defined by Curry [33]. They depend on the molecule/pore radius ratio $\alpha_{k}$, so that, for a fixed pore radius, the diffusion coefficient in the pore decreases as the solute molecule radius increases. This effect results from the viscous layer of fluid at the pore walls, which retards the solute diffusion increasingly with its radius. The relation between the radius of a molecule and its diffusion coefficient in a medium is given by Stokes-Einstein equation (Eq.12).

$$
D=\frac{R T}{6 \pi N \eta r}
$$

where $R$ is the gaz constant, $\mathrm{T}$ is the absolute temperature, $\mathrm{N}$ is the Avogadro number, $\eta$ is the medium viscosity and $\mathrm{r}$ is the molecule radius. The permeability is controlled by the properties of the pore and the vessel or capillary (radius, area, membrane wall thickness $\Delta r$ ), as well as those of the molecule (radius $r$ and diffusion coefficient $D)$. 


\section{Model equations}

Besides some findings of the MID method [6], the volume of each compartment is assumed constant and the vessels are modeled as well-stirred compartments and are filled with the molecule instantaneously. Otherwise, we would need to apply a volume gradient.

The agent propagation is described by the system of differential equations given below, where the variation of the molecular concentration $C(t)$ in each compartment is computed as a function of time.

$$
\begin{aligned}
V_{h a} \frac{d C_{h a}(t)}{d t}= & Q_{h a}^{0} C_{h a}^{0}(t)-S_{h a} \times_{h a} J_{s}(t)-Q_{h a} C_{h a}(t) \\
V_{p v} \frac{d C_{p v}(t)}{d t}= & Q_{p v}^{0} C_{p v}^{0}(t)-S_{p v} \times_{p v} J_{s}(t)-Q_{p v} C_{p v}(t) \\
V_{s i} \frac{d C_{s i}(t)}{d t}= & Q_{h a} C_{h a}(t)+Q_{p v} C_{p v}(t)-S_{s i} \times_{s i} J_{s}(t)-Q_{s i} C_{s i}(t) \\
V_{i l} \frac{d C_{i l}(t)}{d t}= & S_{h a} \times_{h a} J_{s}(t)+S_{p v} \times_{p v} J_{s}(t)+S_{h v} \times_{h v} J_{s}(t) \\
& +S_{s i} \times_{s i} J_{s}(t)-Q_{L} C_{i l}(t) \\
V_{h v} \frac{d C_{h v}(t)}{d t}= & Q_{s i} C_{s i}(t)-S_{h v} \times_{h v} J_{s}(t)-Q_{h v} C_{h v}(t)
\end{aligned}
$$

The term subscripts are in concordance with each compartment initials (ha, pv, si, il, hv, stand for hepatic arteriole, portal venule, sinusoids, interstitial liquid and hepatic venule respectively). Based on the hypothesis assumed for the volumes and a knowledge of the flows at the input to the model, the mass conservation law can be applied to the flow in each compartment, while specifying that the output flow equals the input flow (Eq.18 to Eq.21).

$$
\begin{aligned}
Q_{h a} & =Q_{h a}^{0}-S_{h a} \times_{h a} J_{f} \\
Q_{p v} & =Q_{p v}^{0}-S_{p v} \times_{p v} J_{f} \\
Q_{s i} & =Q_{h a}+Q_{p v}-S_{s i} \times_{s i} J_{f}
\end{aligned}
$$




$$
Q_{h v}=Q_{s i}-S_{h v} \times_{h v} J_{f}
$$

High interstitial pressures and low lymphatic outlet pressures can lead to an evacuation of the interstitium through the lymphatics, resulting in a lymph flow $Q_{L}$ (Eq.22), where $K_{L}$ is the lymphatic conductance.

$$
Q_{L}=\left\{\begin{aligned}
K_{L}\left(p_{i l}-p_{L}\right) & \text { for } p_{i l}>p_{L} \\
0 & \text { for } p_{i l} \leq p_{L}
\end{aligned}\right.
$$

However, in our simplifications, compartments volumes are considered as constant. Fluid balance (Eq.23) thus requires that the lymphatic flow is defined as the sum of the net transendothelial fluxes (Eq.24).

$$
\begin{gathered}
Q_{h a}^{0}+Q_{p v}^{0}=Q_{h v}+Q_{L} \\
Q_{L}=S_{h a} \times_{h a} J_{f}+S_{p v} \times_{p v} J_{f}+S_{s i} \times_{s i} J_{f}+S_{h v} \times_{h v} J_{f}
\end{gathered}
$$

\section{Numerical method and simulation procedure}

The volumes, areas and pressures of all compartments are fixed, except for the pressures in the interstitial fluid whose values can be modified. Assuming that the global volume of a healthy adult liver is almost 1350mL [34] and that the whole organ is made up of approximately one million lobules, we can obtain the volume of a functional unit. Inside one unit, the vessels are modeled as cylinders of equal length. Therefore, the volumes of the arteriole and venule compartments depend on their radii and lengths, whose values are given by [35] and [36], while the volumes of interstitial and sinusoids compartments are based on the proportion of each material in the liver $(10,6 \%$ of sinusoids and $4,9 \%$ of interstitial space), as given by [37]. For the vessels, the areas $S$ are computed from their volumes and diameters, while the area of the sinusoidal compartment is given by the average area of one sinusoid 
[38] and the total number of sinusoids in the liver [36]. Hydrostatic and osmotic pressures in the vessels were taken from [32], while the values for the sinusoids and the interstitial fluid are those given in [39]. In the normal case, the vessels (arterioles and venules) are assumed to be impermeable, and consequently have no pores and no fenestrations on their membranes.

The system (Eq.13 to Eq.17) is computed numerically under MS Visual C++ 6.0, with an ordinary differential equation (ODE) solver based on a fourth-order RungeKutta method. The molecular concentration is measured in each compartment for different physiological parameters, and its variation plotted as a function of time. Comparable results were obtained when programming with Matlab, as well as with JSIM, a modeling environment developed by the National Simulation Resource for Circulatory Mass Transport (http://nsr.bioeng.washington.edu).

In the first series of validation tests, a constant concentration $(0.5 \mathrm{mM})$ is chosen for the inputs $C_{h a}^{0}(t)$ and $C_{p v}^{0}(t)$, in order to observe the steady-state behavior of the model. The reference arterial and portal inflows are $4.16 \mathrm{~cm}^{3} \cdot \mathrm{s}^{-1}$ and $21.8 \mathrm{~cm}^{3} \cdot \mathrm{s}^{-1}$ (Table 1), respectively [16] [40]. These values are divided by the total number of lobules to obtain the inflows in the terminal vessels, which thus form the model inputs. Here, only the capillaries are permeable. These values can be modified in different tests with arbitrary values. Gd-DOTA is chosen as the reference molecule. This molecule is modeled as a simple sphere. In our model, both the radius and diffusion coefficient of Gd-DOTA $\left(r=0.45 n m\right.$ and $\left.D=47 . e^{-7} \mathrm{~cm}^{2} \cdot \mathrm{s}^{-1}[26]\right)$, have an influence on its concentration variation. In [26], the radius of Gd-DOTA was computed from Eq.12 for a measured diffusion coefficient under given conditions. The diffusion coefficient of the two other molecules were thus deducted from their known radius, with the same equation parameters. After this stage of validation, the model can be used to simulate real pathological situations, which is the main purpose of the present approach. With this aim in view, we use the data provided by dynamic contrast enhanced relaxometry to extract curves describing the time- 
evolution of contrast agent concentration in the terminal branches of the hepatic artery and portal vein. Such quantitative method is described in [41]. Here, the procedure is carried out on a sequence of successive MR images of the liver, after a bolus injection of $0.1 \mathrm{mmol} . \mathrm{kg}^{-1}$ dose of Gd-DOTA. Since these data are made up only of a series of points, we require an interpolation step before solving the differential equations system. The "PCHIP" interpolation method (Piecewise Cubic Hermite Interpolating Polynomial [42]) is preferred to cubic spline interpolation in this case, because of the steep slope of the aortic function. 


\section{RESULTS AND DISCUSSION}

Several tests were conducted to evaluate the coherence of the model, and also to simulate pathological cases. In the first series of tests (Fig.5 to 7), the coherence was verified through a steady-state study.

We first simulated variations in the inflows (Fig.5). The example given here involves a decrease in the b) arterial and c) portal supplies. The curves on Fig.5.a) represent the reference case: all the following "steady-state" results were obtained under the same conditions unless notification. Variations in the amount of inflow play an important role in the compartment filling speed, as seen on Fig.5.b) and c), particularly when the portal supply is decreased, as its value is normally much more important. In fact, as a reference condition, the vessels (arterioles and venules) are assumed to be impermeable, which implies, according to the mass conservation law, that all the solute coming into the arteriole and portal venule goes to the sinusoids. Flows only affect the speed, as illustrated on the graph showing that final concentrations in all the compartments reach the maximum value corresponding to the injected solute concentration. The permeability of the capillaries allows the contrast agent concentration to be in equilibrium between the sinuoidal compartment and the interstitial fluid.

In the next step, we tested the influence of each of the two parameters defining the molecule, under normal conditions. In Fig.6 the radius is increased from a) to c). Values given here correspond to a) $r_{G d-D O T A}$, b) $r_{P 792}$ and c) $r_{S H U-555-A}$. Results on Fig. 7 show the effect on the concentrations, of decreasing the diffusion coefficient from a) $D_{G d-D O T A}$, to c) $D_{S H U-555-A}$. From the results presented on Fig.6 and 7 , we conclude that the solute concentration evolution depends strongly on the radius and diffusion coefficient of the molecule. Indeed, an increase in the solute radius $\left(r_{G d-D O T A}<r_{P 792}<r_{S H U-555-A}\right)$ leads to a decreased concentration in the interstitial fluid, due to the decreased number of molecules able to cross the membrane of the sinusoids (Fig.6). However, this difference is not measurable on Fig.6.a) and 
b), where the radius was approximately multiplied by five. At this level, molecules are all able to cross all kinds of pores rapidly. Only a decreased diffusion coefficient in the case of P792 molecule has a real influence on the interstitial concentration (Fig.7.b). In the case of the SPIO agent (Fig.6.c and 7.c), fenestrations are the unique path for the molecule. Consequently, its circulation from the sinusoids to the interstitium is more difficult. Here the radius alone already has a strong influence (Fig.6). The large size of SHU-555-A, combined to its low diffusion coefficient leads to a decreased concentration in the interstitium. In fact, these two properties of the molecule generate opposite reactions, as they are inversely proportional. The parameter $D$ acts as a proportionality factor affecting the sinusoid membrane permeability (see Eq.9), in such a way that decreasing $D$ leads to a decrease in the interstitial molecular concentration. The impact of varying the solute diffusion coefficient $(D)$ differs from that observed when varying the molecular radius. In fact, the molecular radius is directly related to the ratio of solute to pore radius $\alpha$ (Eq.11). This ratio varies inversely with the growth in permeability and osmotic reflection coefficient (Eq.9, 10), and then rises with the solute flux (Eq.3).

In the following results (Fig.8), simulated curves are presented for the three types of extracellular molecules (a) Gd-DOTA, b) P792, c) SHU-555-A). Gd-DOTA is currently used in clinical routine and is thus presented here as the reference molecule. P792 was chosen as an example of a recently developped macromolecular blood pool agent, while SHU-555-A, which has interesting because very different characteristics, has been proved useful in the detection of HCC [28]. Different stages in the development of a HCC (HepatoCellular Carcinoma) in the liver were simulated and the main results are presented in Figs.8.2 to 8.4. At the outset, the liver is characterized by an initial high arterial concentration peak (green curve), followed by transit of the molecule through the portal venule (red curve). In a healthy liver (Fig.8.1), sinusoids (blue curve) are mostly supplied by the portal venule, and communicate directly with the hepatic venule (yellow curve). The molecular concentration in 
the sinusoids then becomes closely similar to the portal concentration, except at the outset when only the arteriole is supplied. Sinusoidal pores and fenestrations allow solute communication with the interstitial fluid (black curve), where the concentration decreases as the molecule size increases and consequently as its diffusion coefficient decreases, especially for larger molecules as SPIO (Fig.8.1.c).

The high-grade dysplastic nodule (DNh) is characterized by a decrease in the arterial and portal supply, an increasing permeability of the vessels (arterioles and venules) - some small and large pores appear on the vessel membrane but no fenestrations are present - and a decreased number of fenestrations in the capillary membrane (Fig.8.2). One direct consequence of weaker portal and arterial inflows is that concentrations in sinusoids, interstitial fluid and hepatic venule are lower than those obtained in the "normal situation". The behavior of both Gd-DOTA and P792 molecules is very similar (Fig.8.2.a) and b)) while the area of communication with the interstitial fluid has more effect on SHU-555-A (Fig.8.2.c)).

In the case of the medium-differentiated carninoma $(\mathrm{HCCm})$, neoangiogenesis which corresponds to the birth of new vessels on the arterial tree, leads to an increase of the arterial supply inversely with the portal one in the tumoral region (see Fig.1). Fenestrations disappear from the sinusoids (Fig.8.3), according to a capillarization phenomenon, also observed in liver cirrhosis [43] [44]. The disappearance of fenestrated pores at this stage of the pathology will result in an increased sinusoidal concentration of the solute, which encounters difficulties in crossing the capillary wall, and a decreased concentration in the interstitial fluid, where it is almost equal to zero for larger molecules (Fig.8.3.b)). This curve normally reaches the equilibrium value but few minutes later. However, it is impossible for SPIO molecule to penetrate the interstitial fluid since it's diameter is higher than those of the pores (Fig.8.3.c)). Globally, as the portal supply decreases, i.e. as the tumor malignancy becomes more important, the hepatic circulatory system tends to "arterialize", as illustrated by the curves on Fig.8.3 and 8.4 where the maxima approach the arterial 
peak.

A significant growth in the arterial supply, combined with an inhibition of the portal supply, is more particularly characteristic of a poorly-differentiated carcinoma (HCCp) (Fig.8.4). The arterialization thus becomes even more evident. The presence of small and large pores in arterioles and venules allows some exchanges with the interstitial fluid. This typical effect is expressed here by a lower but non-zero molecular concentration in the portal venule. As a result, sinusoidal and hence hepatic venule concentrations as well, even if mostly dependent on the arterial supply in this case, are also affected by the portal supply, at least with the Gd-DOTA molecule (Fig.8.4.a)). The same observations as for HCCm curves can be made for the blood pool (P792) and SPIO (SHU-555-A) agents. In fact, the input profiles used in our application (with an early arterial peak) are "Gd-DOTA-specific". More realistic results for larger molecules such as USPIO and SPIO, which have a longer residence time in the vessels, will necessitate to take into account well corresponding profiles in future studies. Moreover, the diffusion phenomena in the large vessels until the micro-vessels would tend to flatten concentration profiles used as inputs in our compartmental model. Injection profiles at the entry of the aorta should then be different than those at the level of the arteriole. 


\section{CONCLUSION AND FUTURE PROSPECTS}

A model of molecular exchanges in hepatic microvascularization, integrating functional parameters such as permeability, flux or fenestrations number and size, that vary with the tumor development, is presented in this paper. The coherence of this model was verified under particular conditions of simulation (steady-state) for three kinds of contrast agent molecule. Even if the proton relaxivities $r_{1}$ and $r_{2}$ of a contrast agent are influenced by several parameters (including the contrast agent composition and in the case of Gadolinium, the interactions of the Gd(III) ion with the inner sphere and outer sphere water protons), we decided to consider these parameters constant in our study, and to characterize the molecule by its main physicochemical properties. Modification of tissue water (bound vs. free water ratio, correlation times, etc) during a liver disease evolution is also considered as constant in the model.

Moreover, the model was applied to the modeling of different stages of liver carcinogenesis, from healthy case to Hepatocellular Carcinoma. The simulated concentration vs. time curves appear to be in agreement with known modifications in the liver related to hepatocarcinogenesis, as seen, for example, in arterialization phenomena. However, before using our model to predict the behavior of new contrast agents, an other validation step will have to be done, by comparing our results to in-vivo data. Nevertheless, these data are difficult to obtain in the human liver: only few research teams have published experimental curves [45]. On the contrary, more studies have been led on the rat or on the rabbit [46] [47]. Further modifications are also envisaged to integrate hepatic cell and bile duct compartments in the model. Such evolution should allow us to simulate excretion and active transport, and thus lead to a more realistic and generic type of model. It would also be interesting to add a phagocyte compartment for contrast agents (such as USPIO or SPIO molecules) that are associated with phagocytosis.

Modeling transport processes is of great interest since we intend to use this model, 
not only to test the behavior of new contrast agents for MRI and other imaging techniques, but also to simulate the pharmacokinetics of therapeutic molecules in the hepatic parenchyma.

Furthermore, one of our goals in future work is to couple this pharmacokinetic model with a macroscopic model of the liver [48] [24], in order to simulate tumor images and determine image parameters related to tumor development. In this future study on MR simulation, we will take into account not only the MR sequence parameters $\left(T_{R}, T_{E}\right.$, flip angle, etc) but also $r_{1}$ and $r_{2}$ as the major NMR characteristics of the contrast agent.

\section{Acknowledgements}

The authors wish to acknowledge the reviewers for their valuable and constructive comments that improved the quality of the paper. P.A. Eliat is supported by a financial grant of Region Bretagne OUEST-genopole ${ }^{\circledR}$. The work was undertaken in close collaboration with COST B21. M.S.N. Carpenter post-edited the English style. 


\section{References}

[1] Peters AM. Fundamentals of tracer kinetics for radiologists. The British Journal of Radiology 1998; 71:1116-1129.

[2] Van Beers BE, Leconte I, Materne R, Smith AM, Jamart J, Horsmans Y. Hepatic perfusion parameters in chronic liver disease: dynamic CT measurements correlated with disease severity. Am. J. Roentgen. 2001; 176:667-673.

[3] Jain RK. Delivery of molecular and cellular medicine to solid tumors. Advanced Drug Delivery Reviews 2001; 46:149-168.

[4] Karabulut N., Nevra E. Contrast agents used in MR imaging of the liver. Diagn. Interv. Radiol. 2006; 12:22-30.

[5] Matsui O. Detection and characterization of small hepatocellular carcinoma. Journal of Gastroenterology and Hepatology 2004; 19:S266-S269.

[6] Goresky CA. A linear method for determining liver sinusoidal and extravascular volumes. Am. J. Physiol. 1963; 204:626-640.

[7] Bassingthwaighte JM and Goresky CA. Modeling in the analysis of solute and water exchange in the microvasculature. Handbook of Physiology, Sect. 2, The Cardiovascular System. Vol IV, The Microcirculation. ed. Renkin E.M. and Michel C.C. Bethesda, M.D.: Am. Physiol. Soc. 1984; 549-626.

[8] Roberts MS and Rowland M. A dispersion model of hepatic elimination. 1: Formulation of the model and bolus considerations. J. Pharmaco. Biopharm. $1986 ; 14: 227-260$.

[9] Tirona R, Schwab AJ, Geng W, Pang KS. Hepatic clearance models: comparison of the dispersion and Goresky models in outflow dilution profiles from multiple indicator rat liver studies. Drug Metab. Dispos. 1998; 26:465-475. 
[10] Kety SS and Schmidt CF. The nitrous oxide method for the quantitative determination of cerebral blood flow in man: theory, procedure and normal values. J. Nucl. Med. 1948; 32:1432-1438.

[11] Taniguchi H, Oguro A, Takeuchi K, Miyata K, Takahashi T, Inaba T, Nakahashi H. Difference in regional hepatic blood flow in liver segments-non-invasive measurement of regional hepatic arterial and portal blood flow in human by positron emission tomography with $H_{2}(15)$ O. Ann. Nucl. Med. 1993; 7:141-145.

[12] Taniguchi H, Oguro A, Koyama H, Masuyama M, Takahashi T. Analysis of models for quantification of arterial and portal blood flow in the human liver using PET. J. Comp. Assist. Tomogr. 1996; 20:135-144.

[13] Ziegler SI, Haberkorn U, Byrne H, Tong C, Schosser R, Krieter H, Kaja S, Richolt JA, Lammertsma AA, Price P. Measurement of liver blood flow using oxygen-15 labelled water and dynamic positron emission tomography: limitations of model description. Eur. J. Nucl. Med. 1996; 23:169-177.

[14] Munk OL, Bass L, Roelsgaard K, Bender D, Hansen SB, Keiding S. Liver kinetics of glucose analogs measured in pigs by PET: importance of dual-input blood sampling. J. Nucl. Med. 2001; 42:795-801.

[15] Becker GA, Müller-Schauenburg W, Spilker ME, Machulla H-J, Piert M. A priori identifiability of a one-compartment model with two input functions for liver blood flow measurements. Phys. Med. Biol. 2005; 50:1393-1404.

[16] Materne R, Van Beers BE, Smith AM, Leconte I, Jamart J, Dehoux J-P, Keyeux A, Horsmans Y. Non-invasive quantification of liver perfusion with dynamic computed tomography and a dual-input one-compartmental model. Clinical Science 2000; 99:517-525. 
[17] Anissimov YG and Roberts MS. A compartmental model of hepatic disposition kinetics: 1. model development and application to linear kinetics. J. Pharmacok. Pharmacod. 2002; 29(2):131-156.

[18] Gambhir SS, Hawkins RA, Sung-Cheng H, Hall TR, Busuttil RW, Phelps ME. Tracer kinetic modeling approaches for the quantification of hepatic function with technetium-99m DISIDA and scintigraphy. J. Nucl. Med. 1989; 30(9):15071518.

[19] Bae KT, Heiken JP, Brink JA. Aortic and hepatic peak enhancement at CT: effect of contrast medium injection rate - pharmacokinetic analysis and experimental porcine model. Radiology 1998; 206:455-464.

[20] Bert JL and Pinder KL. Analog simulation of the human microvascular exchange system. Simulation 1982; 39:89-95.

[21] Sarangapani R, Teeguarden J, Plotzke KP, McKim JM, Andersen ME. Doseresponse modeling of cytochrome P450 induction in rats by octamethylcyclotetrasiloxane. Toxicological Sciences 2002; 67:159-172.

[22] Andersen ME, Eklund CR, Mills JJ, Barton HA, Birnbaum LS. A multicompartment geometric model of the liver in relation to regional induction of cytochrome P450s. Toxicol. Appl. Pharm. 1997; 144:135-144.

[23] Kapanen MK, Halavaara JT, Häkkinen A-M. Open four-compartment model in the measurement of liver perfusion. Acad. Radiol. 2005; 12:1542-1550.

[24] Bézy-Wendling J, Kretowski M, Coupe P. Modeling of tumor conspicuity in hepatic CT images: combined compartment and vascular model. IEEE Int. Symp. on Biomedical imaging: Macro to Nano 2004; 1:444-447. 
[25] Kretowski M, Bezy-Wendling J, Coupe P. simulation of biphasic CT findings in hepatic cellular carcinoma by a two-level physiological model. IEEE Transactions on Biomedical Engineering (in print); TBME-00187.

[26] Corot C, Port M, Raynal I, Dencausse A, Schaefer M, Rousseaux O, Simonot C, Devoldere L, Lin J, Foulon M, Bourrinet P, Bonnemain B, Meyer D. Physical, chemical and biological evaluations of P760: a new gadolinium complex characterized by a low rate of interstitial diffusion. Journal of Magnetic Resonance Imaging 2000; 11:182-191.

[27] Vander Elst L, Raynal I, Port M, Tisnès P, Muller RN. In vitro relaxometric and luminescence characterization of P792 (Gadomelitol, Vistarem ${ }^{\circledR}$ ), an efficient and rapid clearance blood pool MRI contrast agent. Eur. J. Inorg. Chem. 2005; $1142-1148$.

[28] Arbab AS, Ichikawa T, Toyama K, Nambu A, Ohsawa S., Kumagai H, Aikawa Y. Detection of hepatocellular carcinoma and its metastases with various pulse sequences using superparamagnetic iron oxide (SHU-555-A). Abdominal Imaging 2000; 25:151-158.

[29] Kellen MR, Bassingthwaighte JB. An integrative model of coupled water and solute exchange in the heart. Am. J. Physiol. Heart. Circ. Physiol. 2003; 285:13031316.

[30] Jain RK. Transport of molecules across tumor vasculature. Cancer and Metastasis Review 1987; 6:559-593.

[31] Patlak CS, Goldstein DA, Hoffman JF. The flow of solute and sovent across a two-membrane system. J. Theor. Biol. 1963; 5:426-442.

[32] Arias, Irwin M. ed. The liver: biology and pathobiology. Lippincott Williams and Wilkins 2001. 
[33] Curry FE. Mechanics and thermodynamics of transcapillary exchange. Handbook of Physiology, The Cardivascular System. Microcirculation. Bethesda, MD: Am. Physiol. Soc. 1984; sect. 2, vol. IV, pt.1, chapt. 8, p. 309-374.

[34] Hausken T, Leotta DF, Helton S, Kowdley KV, Goldman B, Vaezy S, Bolson EL, Sheehan FH, Martin RW. Estimation of the human liver volume and configuration using three-dimensional ultrasonography: effect of a high-caloric liquid meal. Ultrasound in Med. Biol. 1988; 24(9):1357-1367.

[35] Crawford AR, Lin X-Z, Crawford JM. The normal adult human liver biopsy: a quantitative reference standard. Hepatology 1998; 28:323-331.

[36] Kerdok AE and Howe RD. A physical basis for a two time constant constitutive model for liver. Summer Bioengineering Conference June 2005; 22-26.

[37] Kompella UB. Protein Drug Delivery. Biopharmaceutical Drug Design and Development, Humana Press 1999; chapt.10, p. 239.

[38] Onori P, Morini S, Franchitto A, Sferra R, Alvaro D, Gaudio E. Hepatic microvascular features in experimental cirrhosis: a structural and morphometrical study in CQ-treated rats. Journal of Hepatology 2000; 33:55-563.

[39] Heldin C-H, Rubin K, Pietras K, Ostman A. High intestitial fluid pressure An obstacle in cancer therapy. Nature 2004; 4:806-813.

[40] Greenway CV and Stark RD. Hepatic vascular bed. Physiological Reviews 1971; $51: 23-52$.

[41] Eliat P-A, Dedieu V, Bertino C, Bouté V, Lacroix J, Constans J-M, de Korvin B, Vincent C, Bailly C, Joffre F, de Certaines JD, Vincensini D. Magnetic resonance imaging contrast-enhanced relaxometry of breast tumors: an MRI multicenter investigation concerning 100 patients. Magn. Res. Im. 2004; 22:475481. 
[42] Fritsch FN and Carlson RE. Monotone Piecewise Cubic Interpolation. SIAM J. Numerical Analysis 1980; 17:238-246.

[43] Huet P-M, Goresky CA, Villeneuve J-P, Marleau D. Assessment of liver microcirculation in human cirrhosis. J. Clin. Invest. 1982; 70(6):1234-1244.

[44] Reichen J, Egger B, Ohara N, Zeltner TB, Zysset T, Zimmermann A. Determinants of hepatic function in liver cirrhosis in the rat. Multivariate analysis. J. Clin. Invest. 1988; 82(6):2069-2076.

[45] Peeters F, Annet L, Hermoye L, Van Beers BE. Inflow correction of hepatic perfusion measurements using T1-weighted, fast gradient-echo, contrast-enhanced MRI. Magn. Res. Med. 2004; 51(4):710-717.

[46] Van Beers BE, Materne R, Annet L, Hermoye L, Sempoux C, Peeters F, Smith AM, Jamart J, Horsmans Y. Capillarization of the sinusoids in liver fibrosis: noninvasive assessment with contrast-enhanced MRI in the rabbit. Magn. Res. Med. 2003; 49(4):692-699.

[47] Van Beers BE, Sempoux C, Materne R, Delos M, Smith AM. Biodistribution of ultrasmall iron oxide particles in the rat liver. J. Magn. Res. Im. 2001; $13(4): 594-599$.

[48] Kretowski M, Rolland Y, Bézy-Wendling J, Coatrieux J-L. Physiologically based modeling of 3-D vascular network and CT scan angiography. IEEE Transactions on Medical Imaging 2003; 22:248-257. 


\section{Captions}

Figure 1: Evolution of blood supply during hepatocarcinogenesis (RN : Regenerative Nodule, DNl,h : low, high grade Dysplastic Nodule, HCCw,m,p : well, moderate, and poorly differentiated $H C C$ ) from [5].

Figure 2: Structure of the hepatic lobule.

Figure 3: Five compartments model of the hepatic microvascular transport of molecules. Symbols: see table 1. Indexes ha, pv, si, hv and il represent the hepatic arteriole, portal venule, sinusoids, hepatic venule and interstitial fluid compartments respectively; $L$ represents the lymph. 
Figure 4: Osmosis: two solutions of different concentration are open at the atmosphere but separated by a semi-permeable membrane which is permeable to the smaller solvent molecules but not to the larger solute molecules. The solvent will thus tend to diffuse across the membrane from the less concentrated to the more concentrated solution. The osmotic pressure in the solution contained in the chamber $A$ is equal to the equilibrium pressure difference across the membrane.

Figure 5: Evolution of a molecule concentration vs. time, in the five compartments of the compartmental model. Influence of the inflows: a) Reference case: $Q_{h a}^{0}=4.16 \mathrm{~cm}^{3} \cdot \mathrm{s}^{-1}$ and $Q_{p v}^{0}=21.8 \mathrm{~cm}^{3} \cdot \mathrm{s}^{-1}$, permeable sinusoids $\left(r_{s p}=6 \mathrm{~nm}\right.$, $d_{s p}=12.5 n b_{s p} \cdot \mu m^{-2} ; r_{l p}=27.5 n m, d_{l p}=0.05 n b_{l p} . \mu m^{-2} ; r_{f}=75 n m, d_{f}=$ $11 \mathrm{nb}_{f} \cdot \mu \mathrm{m}^{-2}$ ) [30][32] and impermeable vessels. Molecule Gd-DOTA: $r=0.9 \mathrm{~nm}$ and $D=70 . e^{-7} \mathrm{~cm}^{2} . \mathrm{s}^{-1}$. b) QhaO is decreased compared to a: $Q_{h a}^{0}=2 \mathrm{~cm}^{3} \cdot \mathrm{s}^{-1}$ and $Q_{p v}^{0}=21.8 \mathrm{~cm}^{3} \cdot \mathrm{s}^{-1}$. c) Qpv0 is decreased compared to $a: Q_{h a}^{0}=4.16 \mathrm{~cm}^{3} \cdot \mathrm{s}^{-1}$ and $Q_{p v}^{0}=10 \mathrm{~cm}^{3} \cdot \mathrm{s}^{-1}$. (Note: Sinusoids, hepatic venule and interstitial fluid concentration curves are superposed).

Figure 6: Evolution of a molecule concentration vs. time, in the five compartments of the compartmental model. Influence of the molecule radius: $a$ ) Reference case: $Q_{h a}^{0}=4.16 \mathrm{~cm}^{3} \cdot \mathrm{s}^{-1}$ and $Q_{p v}^{0}=21.8 \mathrm{~cm}^{3} \cdot \mathrm{s}^{-1}$, permeable sinusoids $\left(r_{s p}=6 n m, d_{s p}=12.5 n b_{s p} . \mu m^{-2} ; r_{l p}=27.5 n m, d_{l p}=0.05 n b_{l p} . \mu m^{-2}\right.$; $r_{f}=75 \mathrm{~nm}, d_{f}=11 \mathrm{nb}_{f} \cdot \mu \mathrm{m}^{-2}$ ) [30][32] and impermeable vessels. Molecule GdDOTA: $r=0.9 \mathrm{~nm}$ and $D=47 . e^{-7} \mathrm{~cm}^{2} . \mathrm{s}^{-1}$. b) $r=2.5 \mathrm{~nm}$. c) $r=31 \mathrm{~nm}$.

Figure 7: Evolution of a molecule concentration vs. time, in the five compartments of the compartmental model. Influence of the molecule diffusion coefficient: a) Reference case: $Q_{h a}^{0}=4.16 \mathrm{~cm}^{3} \cdot \mathrm{s}^{-1}$ and $Q_{p v}^{0}=21.8 \mathrm{~cm}^{3} \cdot \mathrm{s}^{-1}$, permeable sinusoids $\left(r_{s p}=6 n m, d_{s p}=12.5 n b_{s p} . \mu m^{-2} ; r_{l p}=27.5 n m, d_{l p}=0.05 n b_{l p} \cdot \mu m^{-2} ; r_{f}=75 n m\right.$, $d_{f}=11 n b_{f} \cdot \mu m^{-2}$ ) [30][32] and impermeable vessels. Molecule Gd-DOTA: $r=$ $0.9 \mathrm{~nm}$ and $D=47 . e^{-7} \mathrm{~cm}^{2} . \mathrm{s}^{-1}$. b) $D=8.4 e^{-7} \mathrm{~cm}^{2} . \mathrm{s}^{-1}$. c) $D=68 e^{-9} \mathrm{~cm}^{2} . \mathrm{s}^{-1}$. 
Figure 8: Evolution of a molecule concentration vs. time, in the five compartments of the compartmental model. 8.1. Normal liver (Reference case): $Q_{h a}^{0}=4.16 \mathrm{~cm}^{3} \cdot \mathrm{s}^{-1}$ and $Q_{p v}^{0}=21.8 \mathrm{~cm}^{3} \cdot \mathrm{s}^{-1}$, permeable sinusoids $\left(r_{s p}=6 \mathrm{~nm}\right.$, $d_{s p}=12.5 n b_{s p} \cdot \mu m^{-2} ; r_{l p}=27.5 n m, d_{l p}=0.05 n b_{l p} . \mu m^{-2} ; r_{f}=75 n m, d_{f}=$ $11 \mathrm{nb}_{f} \cdot \mu \mathrm{m}^{-2}$ ) [30][32] and impermeable vessels. 8.2. Dysplastic Nodule (DNh): $Q_{h a}^{0}=2.74 \mathrm{~cm}^{3} \cdot \mathrm{s}^{-1}$ and $Q_{p v}^{0}=9.54 \mathrm{~cm}^{3} \cdot \mathrm{s}^{-1}$, permeable sinusoids $\left(r_{s p}=6 \mathrm{~nm}, d_{s p}=\right.$ $\left.12.5 n b_{s p} \cdot \mu m^{-2} ; r_{l p}=27.5 n m, d_{l p}=0.05 n b_{l p} \cdot \mu m^{-2} ; r_{f}=75 n m, d_{f}=5 n b_{f} \cdot \mu m^{-2}\right)$ and vessels $\left(r_{s p}=6 n m, d_{s p}=6 n b_{s p} . \mu m^{-2} ; r_{l p}=27.5 n m, d_{l p}=1 / 20 n b_{l p} . \mu m^{-2}\right)$. Both sides: a) Molecule Gd-DOTA: $r=0.45 \mathrm{~nm}$ and $D=47 . e^{-7} \mathrm{~cm}^{2} \cdot \mathrm{s}^{-1}$ (Note: hepatic venule, sinusoids and interstitium concentration curves are almost superposed. b) Molecule P792: $r=2.5 \mathrm{~nm}$ and $D=8.4 e^{-7} \mathrm{~cm}^{2} . \mathrm{s}^{-1}$. c) Molecule SHU555-A: $r=31 \mathrm{~nm}$ and $D=68 . e^{-9} \mathrm{~cm}^{2} \cdot \mathrm{s}^{-1}$ (Note: hepatic venule and sinusoids concentration curves are superposed on $b$ and $c)$.

Figure 8-continued: 8.3. Medium Differentiated Hepatocellular Carcinoma $(\mathbf{H C C m}): Q_{h a}^{0}=6.24 \mathrm{~cm}^{3} \cdot \mathrm{s}^{-1}$ and $Q_{p v}^{0}=4.09 \mathrm{~cm}^{3} \cdot \mathrm{s}^{-1}$, no fenestrations in sinusoids $\left(r_{s p}=6 n m, d_{s p}=12.5 n b_{s p} . \mu m^{-2} ; r_{l p}=27.5 n m, d_{l p}=0.05 n b_{l p} . \mu m^{-2}\right)$ and permeable vessels $\left(r_{s p}=6 n m, d_{s p}=6 n b_{s p} . \mu m^{-2} ; r_{l p}=27.5 n m, d_{l p}=0.05 n b_{l p} . \mu m^{-2}\right)$. 8.4. Poorly Differentiated Hepatocellular Carcinoma (HCCp): $Q_{h a}^{0}=$ $10.15 \mathrm{~cm}^{3} \cdot \mathrm{s}^{-1}$ and $Q_{p v}^{0}=0 \mathrm{~cm}^{3} \cdot \mathrm{s}^{-1}$ no fenestrations in sinusoids $\left(r_{s p}=6 \mathrm{~nm}\right.$, $\left.d_{s p}=12.5 n b_{s p} \cdot \mu m^{-2} ; r_{l p}=27.5 n m, d_{l p}=0.05 n b_{l p} . \mu m^{-2}\right)$ and permeable vessels $\left(r_{s p}=6 n m, d_{s p}=6 n b_{s p} . \mu m^{-2} ; r_{l p}=27.5 n m, d_{l p}=0.05 n b_{l p} . \mu m^{-2}\right)$. Both sides: a) Molecule Gd-DOTA: $r=0.45 \mathrm{~nm}$ and $D=47 . e^{-7} \mathrm{~cm}^{2} \cdot \mathrm{s}^{-1}$. b) Molecule Pr92: $r=2.5 \mathrm{~nm}$ and $D=8.4 \mathrm{e}^{-7} \mathrm{~cm}^{2} \cdot \mathrm{s}^{-1}$. c) Molecule SHU-555-A: $r=31 \mathrm{~nm}$ and $D=68 e^{-9} \mathrm{~cm}^{2} \cdot \mathrm{s}^{-1}$. (Note: hepatic venule and sinusoids concentration curves are superposed). 
Table 


\begin{tabular}{|c|c|c|c|}
\hline & Definition & Value and Unit & Reference \\
\hline$C_{h a}^{0}$ & Initial concentration in hepatic artery & $0.5 \mathrm{mM}$ & depends on the injection profile \\
\hline$C_{p v}^{0}$ & Initial concentration in portal vein & $0.5 \mathrm{mM}$ & depends on the injection profile \\
\hline$Q_{h a}^{0}$ & Arterial inflow & $4.16 \mathrm{~cm}^{3} \cdot \mathrm{s}^{-1}$ & {$[16][40]$} \\
\hline$Q_{p v}^{0}$ & Portal inflow & $21.8 \mathrm{~cm}^{3} \cdot \mathrm{s}^{-1}$ & {$[16][40]$} \\
\hline$r$ & Molecule radius & $0.45 \mathrm{~nm}$ & {$[26]$} \\
\hline$D$ & Molecule diffusion coefficient & $70 \times 10^{-7} \mathrm{~cm}^{2} \cdot \mathrm{s}^{-1}$ & {$[26]$} \\
\hline$r_{h a}$ & Hepatic arteriole radius & $6 \mu m$ & {$[35]$} \\
\hline$r_{p v}$ & Portal venule radius & $17.5 \mu \mathrm{m}$ & {$[35]$} \\
\hline$r_{h v}$ & Hepatic venule radius & $33 \mu m$ & {$[35]$} \\
\hline$l_{h a, p v, h}$ & Arteriole, venule length & $4 m m$ & {$[36]$} \\
\hline$V_{h a}$ & ha compartment Volume & $0.45 \times 10^{-6} \mathrm{~cm}^{3}$ & {$[35][36]$} \\
\hline$V_{p v}$ & pv compartment Volume & $3.84 \times 10^{-6} \mathrm{~cm}^{3}$ & {$[35][36]$} \\
\hline$V_{s i}$ & si compartment Volume & $143 \times 10^{-6} \mathrm{~cm}^{3}$ & {$[37]$} \\
\hline$V_{i l}$ & il compartment Volume & $66 \times 10^{-6} \mathrm{~cm}^{3}$ & {$[37]$} \\
\hline$V_{h v}$ & hv compartment Volume & $13.6 \times 10^{-6} \mathrm{~cm}^{3}$ & {$[35][36]$} \\
\hline$S_{h a}$ & ha compartment Area & $15 \times 10^{-4} \mathrm{~cm}^{2}$ & {$[35][36]$} \\
\hline$S_{p v}$ & pv compartment Area & $44 \times 10^{-4} \mathrm{~cm}^{2}$ & {$[35][36]$} \\
\hline$S_{s i}$ & si compartment Area & $28.6 \times 10^{-4} \mathrm{~cm}^{2}$ & {$[38][36]$} \\
\hline$S_{h v}$ & hv compartment Area & $83 \times 10^{-4} \mathrm{~cm}^{2}$ & {$[35]$} \\
\hline$p_{h a}$ & Hydrostatic pressure in the arteriole & $16 m m H g$ & {$[32]$} \\
\hline$p_{p v, h v}$ & Hydrostatic pressure in the venule & $5 \mathrm{mmHg}$ & {$[32]$} \\
\hline$p_{s i}$ & Hydrostatic pressure in the capillary & $20 \mathrm{mmHg}$ & {$[39]$} \\
\hline$p_{i l}$ & Hydrostatic pressure in the interstitial fluid & $-3 m m H g$ & {$[39]$} \\
\hline$\Pi_{v}$ & Osmotic pressure in the vessel or capillary & $28 \mathrm{mmHg}$ & {$[39]$} \\
\hline$\Pi_{i l}$ & Osmotic pressure in the interstitial fluid & $8 m m H g$ & {$[39]$} \\
\hline$\Delta r_{h a}$ & Arteriole wall thickness & $6 \mu m$ & arbitrary value \\
\hline$\Delta r_{p v, h v}$ & Venule wall thickness & $1 \mu m$ & arbitrary value \\
\hline$\Delta r_{s i}$ & Capillary wall thickness & $0.5 \mu m$ & arbitrary value \\
\hline$\eta$ & Perfusate viscosity $\left(37^{\circ}\right)$ & $0.007 \mathrm{~g} . \mathrm{cm}^{-1} \cdot \mathrm{s}^{-1}$ & {$[29]$} \\
\hline$r_{s p}$ & Small pore radius & $6 n m$ & {$[30]$} \\
\hline$r_{l p}$ & Large pore radius & $27.5 \mathrm{~nm}$ & {$[30]$} \\
\hline$r_{f}$ & Fenestration radius & $75 \mathrm{~nm}$ & {$[32]$} \\
\hline$d_{s p}$ & Small pore density & $12.5 n b_{s p \cdot \mu m^{-2}}$ & {$[30]$} \\
\hline$d_{l p}$ & Large pore density & $0.05 n b_{l p} \cdot \mu m^{-2}$ & {$[30]$} \\
\hline$d_{f}$ & Fenestration density & $11 n b_{f} \cdot \mu m^{-2}$ & {$[32]$} \\
\hline
\end{tabular}

Table 1: Terminology and parameter values 


\begin{tabular}{|c|c|c|c|}
\hline & Definition & Value and Unit & Reference \\
\hline$\alpha$ & Ratio of solute radius to pore radius & 0-1 (dimensionless) & computed from Eq.11 \\
\hline $\mathrm{Pe}$ & Péclet number & $0-2$ (dimensionless) & computed from Eq.4 \\
\hline$\Pi_{M}$ & Osmotic pressure of immobile interstitial matrix & $m m H g$ & computed from Eq.2 \\
\hline$M_{i m}$ & Quantity of interstitial matrix proteins & $0.05 g \cdot m L^{-1}$ & {$[29]$} \\
\hline$\psi_{i}$ & Matrix osmotic pressure coefficients & $\begin{array}{c}\psi_{1}=13.5 \\
\psi_{2}=2320 \\
\psi_{3}=1.54 \times 10^{5}\end{array}$ & {$[29]$} \\
\hline${ }_{i} J_{s}^{k}$ & $\begin{array}{l}\text { Solute flux through the } k^{s t} \text { path } \\
\text { from the vessel } i \text { to the interstitial fluid }\end{array}$ & mmol.cm ${ }^{-2} . s^{-1}$ & computed from Eq.3 \\
\hline${ }_{i} J_{f}^{k}$ & $\begin{array}{l}\text { Fluid flux through the } k^{s t} \text { path } \\
\text { from the vessel } i \text { to the interstitial fluid }\end{array}$ & $\mathrm{cm} \cdot \mathrm{s}^{-1}$ & computed from Eq.1 \\
\hline$A_{k}$ & Exchange area related to the $k^{s t}$ path & $\mathrm{cm}^{2}$ & computed from $\left(\Pi r_{k}^{2} d_{k} S\right)$ \\
\hline$L_{k}$ & Hydraulic conductivity related to the $k^{s t}$ path & $c m . s^{-1} \cdot m m H g^{-1}$ & computed from Eq.8 \\
\hline$L_{\text {endo }}$ & Transendothelial hydraulic conductivity & $1.4 \times 10^{-8} \mathrm{~cm} . \mathrm{s}^{-1} \cdot \mathrm{mmHg} \mathrm{g}^{-1}$ & {$[29]$} \\
\hline$P_{k}$ & Permeability through to the $k^{s t}$ path & $\mathrm{cm} . \mathrm{s}^{-1}$ & computed from Eq.9 \\
\hline$\sigma_{k}$ & Osmotic reflection coefficient through the $k^{s t}$ path & dimensionless & computed from Eq.10 \\
\hline$R$ & Gaz constant & $8.33 S I$ & \\
\hline$T$ & Absolute temperature & $300^{\circ} \mathrm{K}$ & \\
\hline$N$ & Avogadro number & $6.10^{23}$ mole $^{-1}$ & \\
\hline$C_{h a}$ & Concentration in hepatic arteriole & $m M$ & \\
\hline$C_{p v}$ & Concentration in portal venule & $m M$ & \\
\hline$C_{s i}$ & Concentration in sinusoids & $m M$ & \\
\hline$C_{i l}$ & Concentration in interstitial fluid & $m M$ & \\
\hline$C_{h v}$ & Concentration in hepatic venule & $m M$ & \\
\hline$Q_{h a}$ & Flow in the hepatic arteriole & $c m^{3} \cdot s^{-1}$ & \\
\hline$Q_{p v}$ & Flow in the portal venule & $\mathrm{cm}^{3} \cdot \mathrm{s}^{-1}$ & \\
\hline$Q_{s i}$ & Flow in the sinusoids & $\mathrm{cm}^{3} \cdot \mathrm{s}^{-1}$ & \\
\hline$Q_{h v}$ & Flow in the hepatic venule & $\mathrm{cm}^{3} \cdot \mathrm{s}^{-1}$ & \\
\hline$Q_{L}$ & Lymph flow & $\mathrm{cm}^{3} \cdot \mathrm{s}^{-1}$ & \\
\hline
\end{tabular}

